\title{
Universal behaviour of the thermoelectric power of composite fermions
}

\author{
B. Tieke ${ }^{\text {a,* }}$, U. Zeitler ${ }^{\text {a }}$, R. Fletcher ${ }^{\text {b }}$, S.A.J. Wiegers ${ }^{\text {a }, ~ A . K . ~ G e i m ~}{ }^{\text {a }}$, J.C. Maan ${ }^{\text {, }}$, \\ M. Henini ${ }^{\mathrm{c}}$ \\ " High Field Magnet Laboratory, University of Nijmegen, 6525 ED Nijmegen, The Netherlands \\ b. Physics Department, Queen's University, Kingston, Ontario, Canada K7L3N6 \\ - Department of Physics, University of Nottingham, Nottingham NG7 2RD, UK
}

Received 21 June 1995; accepted for publication 15 September 1995

\begin{abstract}
Abatract
The thermopowers of high-mobility two-dimensional electron gases at even denominator filling factors $v$ are found to be remarkably similar to each other and also to the thermopower at zero magnetic field. The magnitudes at $v=1 / 2$ and $v=3 / 2$ are identical, regardless of temperature and eloctron density. This also appears to be true at $v=1 / 4$ and $\nu=3 / 4$, with the magnitude of this pair being about two times larger than for the former pair. These results are interpreted in terms of the "zero field" phonon drag thermopower of composite fermions.
\end{abstract}

Keywords: Electrical transport measurements; Gallium arsenide; Heterojunctions; Quantum effects; Semiconductor-semiconductor heterostructures

\section{Introduction}

Recently, a remarkably simple description of the fractional quantum Hall effect (FQHE) has been provided by the concept of composite fermions (CFs) $[1,2]$. These quasi-particles are thought to consist of electrons with an even number $2 m$ of flux quanta attached to them. The external magnetic field $B$ transforms for CFs to an effective field $B^{*}=B-B(1 / 2 m)$, which is zero at particular evendenominator filling factors $v=1 / 2 m$. Fractional occupancies around $v=1 / 2 m$ then appear as normal Landau levels of these new particles in $B^{*}$.

\footnotetext{
* Corresponding author. Fax: + 31243652440 , o-mail: benno@scikun.nl.
}

This model has successfully been applied to a number of experiments including magnetotransport $[3,4]$ and surface acoustic waves [5].

In this paper, we report experimental results on the thermoelectric power (TEP) of a high-mobility 2DEG in high magnetic fields, exhibiting the FQHE. In such an experiment, a temperature gradient is applied to the sample and the voltage between contact probes is measured. Our measurements (Refs. [6,7] and also the present work) have shown that TEP in 2DEGs is dominated by phonon drag down to temperatures $T<200 \mathrm{mK}$ and that the diffusion contribution, which has been observed in a $2 \mathrm{D}$ hole gas for $T<100 \mathrm{mK}$ [8], is negligible in our samples. Phonon drag arises from the fact that the applied temperature gradient creates bulk acoustic phonons, which propagate 
through the substrate from the hot to the cold side of the sample and couple to the electrons. Since the net electric current is zero, an electric field appears to offset the current flow due to the electron-phonon interaction. This electric field is measured as the thermoelectric effect. Therefore, TEP measurements directly probe electronphonon interactions, and, if one considers electrons in the FQHE as $\mathrm{CFs}$, serves as a probe for $\mathrm{CF}$-phonon interaction. Here we focus on the longitudinal part of TEP, i.e. $S_{x x}$.

\section{Experimental}

The experiments were performed in a ${ }^{3} \mathrm{He} /{ }^{\wedge} \mathrm{He}$ dilution refrigerator at $100 \mathrm{mK}<T<1.2 \mathrm{~K}$ up to magnetic fields $B$ of $20 \mathrm{~T}$ using standard techniques $[6,7]$. The thermometry was checked at $B=0$ as well as in high fields by evaluating the thermal conductivity $\lambda$ from the data. $\lambda$ was always found to be in excellent agreement with a $T^{3}$ dependence expected for boundary scattering, and showed no field dependence. The electron density of the sample could be adjusted in the range $1.0-1.9 \times 10^{15} \mathrm{~m}^{-2}$ by illumination with an IR diode. The mobility varied between 60 and $100 \mathrm{~m}^{2} / \mathrm{V} \cdot \mathrm{s}$.

\section{Results and discussion}

Fig. 1 shows $S_{x x}$ at a fixed electron density of $n=1.9 \times 10^{15} \mathrm{~m}^{-2}$ as a function of $B$ at various $T$. Apart from the minima at integer and fractional filling factors $v$ due to mobility gaps in the density of states at the Fermi level (which are observed in TEP as well as in resistivity), $S_{x x}$ shows remarkable differences as compared to $\boldsymbol{R}_{x x}$. We will concentrate on these differences, and in particular on the amplitudes of $S_{x x}$ between the minima, which provide unique information.

TEP has a strong temperature dependence of its absolute magnitude (contrary to resistivity) and, to compare the evolution of the curves at different $T$ in Fig. 1, the lower temperature curves have been scaled to the curve at $\$ 84 \mathrm{mK}$ using the points at $v=1 / 2$ as a reference. After this scaling we observe

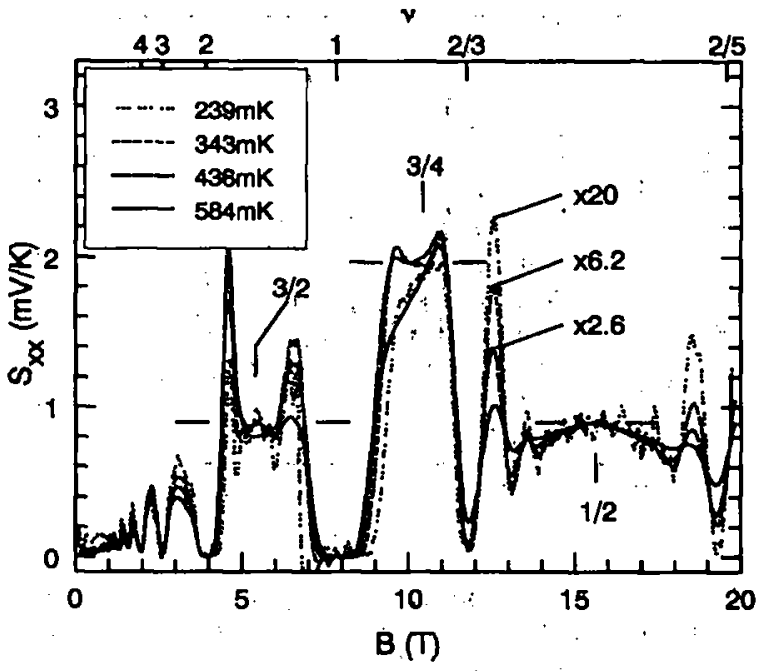

Fig. 1. $S_{x x}$ scaled by its value at $v=1 / 2$ at different temperatures for $n=1.9 \times 10^{15} \mathrm{~m}^{-2}$ as a function of magnetic field $B$.

the striking result that, within experimental accuracy, $S_{x x}(3 / 2)$ (we indicate the filling factor $v=3 / 2$ in parentheses) has the same value as $S_{x x}(1 / 2)$ at all temperatures below $600 \mathrm{mK}$. Moreover; these magnitudes are the same despite their very strong variation "with $T$ (about two orders of magnitude in this temperature range). A similar scaling is observed for $S_{x x}(3 / 4)$, except that here the magnitude is 2.2 times higher than' $S_{x x}(1 / 2)$. Notice that this scaling is unique to the filling factors $v=1 / 2$, $3 / 2$ and $3 / 4$ since the relative amplitudes at other filling factors in the fractional as well as integer quantum Hall regime vary strongly with temperature (e.g. at $v=5 / 8$ and $v=5 / 2$ in Fig. 1).

Fig. 2 shows $S_{x x} / S_{x x}(1 / 2)$ for three eloctron densities at a fixed $T$ as a function of $1 / v$. Notice that the observed equality between $S_{x x}(1 / 2)$ and $S_{x x}(3 / 2)$ also persists for different densities. For $S_{x x}(3 / 4)$, we find that the ratio $S_{x x}(3 / 4) /$ $S_{x x}(1 / 2) \approx 2.2$ temains constant for densities above $n=1.6 \times 10^{15} \mathrm{~m}^{-2}$, which is also the lowest density for which the oscillatory structure in the fractions (e.g. $v=2 / 3$ ) remains well, defined. At the lowest densities, we can also reach $v=1 / 4$ and find $S_{x x}(1 / 4) / S_{x x}(1 / 2) \approx 2.4$, with the ratio decreasing slightly with increasing $n$. This value is very close to 2.2 , the value found for $S_{x x}(3 / 4) / S_{x x}(1 / 2)$, and appears to extrapolate to it when $a$ slight 


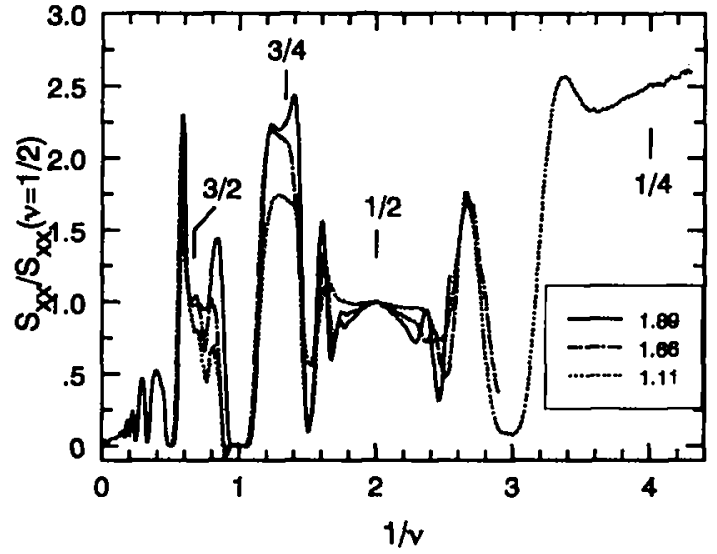

Fig. 2. The scaled thermopower $S_{x x} / S_{x x}(v=1 / 2)$ at $T=440 \mathrm{mK}$ for different electron densities (in units of $10^{15} \mathrm{~m}^{-2}$ ) as a function of the inverse filling factor $1 / v$.

$n$-dependence is taken into account. Furthermore, in previous experiments on lower density samples, $S_{x x}(1 / 4) / S_{x x}(1 / 2)$ has always been found to be in the range $2-3$ [6]. Thus, we observe that within experimental error, $S_{x x}(1 / 4)$ has the same value as $S_{x x}(3 / 4)$.

If we examine the resistivity at comparable temperatures in the same device (Fig. 3) we see no similar correspondence of the absolute resistivities at $v=1 / 2$ and $v=3 / 2\left(R_{x x}(1 / 2) \approx 5 \times R_{x x}(3 / 2)\right)$ or at $v=1 / 4$ and $3 / 4$ (not shown). The observed behaviour is thus unique for TEP. Another notice-

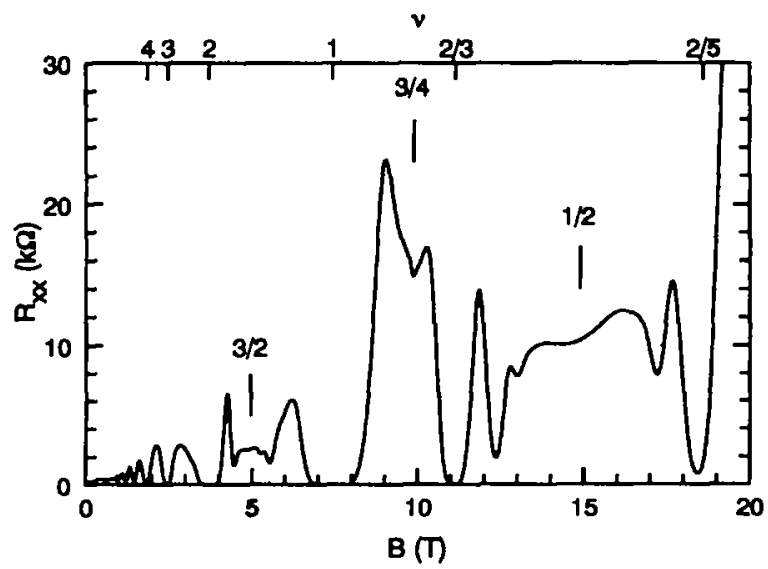

Fig. 3. The resistivity $R_{x x}$ of the same sample $(n=1.8 \times$ $\left.10^{15} \mathrm{~m}^{-2}\right)$ at a comparable temperature $(T=209 \mathrm{mK})$ as a function of magnetic field $B$. able obser/ation is that the symmetry around $v=$ $1 / 2$ is much more pronounced in $S_{x x}$ than in $R_{x x}$.

The temperature dependence of the absolute magnitude of TEP in zero magnetic field $S_{0}$ is found to be described by a $T^{4}$ law at low $T$ (Fig. 4), consistent with phonon drag [9], while no sign of a linear $T$-dependence characteristic for diffusion TEP is observed. $S_{x x}(1 / 2)$ and $S_{0}$ have the same temperature dependence over the whole temperature range, even though their absolute values change by three orders of magnitude. The ratio $S_{x x}(1 / 2) / S_{0}$ is 50 for $n=1.8 \times 10^{11} \mathrm{~cm}^{-2}$. As already indicated in Figs. 1 and 2, the value of $S_{x x}(3 / 2)$ is identical with $S_{x x}(1 / 2)$ below $600 \mathrm{mK}$. The deviation above $T \approx 600 \mathrm{mK}$, marked by the arrow in Fig. 4, coincides with the temperature at which the minima at $v=4 / 3$ and $5 / 3$, i.e. the features of the FQHE around $v=3 / 2$, vanish. $S_{x x}(3 / 4)$ again behaves in a similar way, with its absolute value being roughly two times higher. As is evident in Figs. 1 and 2, $S_{x x}$ at other filling factors (e.g. $v=$ $5 / 8$ and $5 / 2$ ) shows a totally different temperature dependence and does not behave like $S_{0}$.

Phonon drag TEP of electrons in zero magnetic

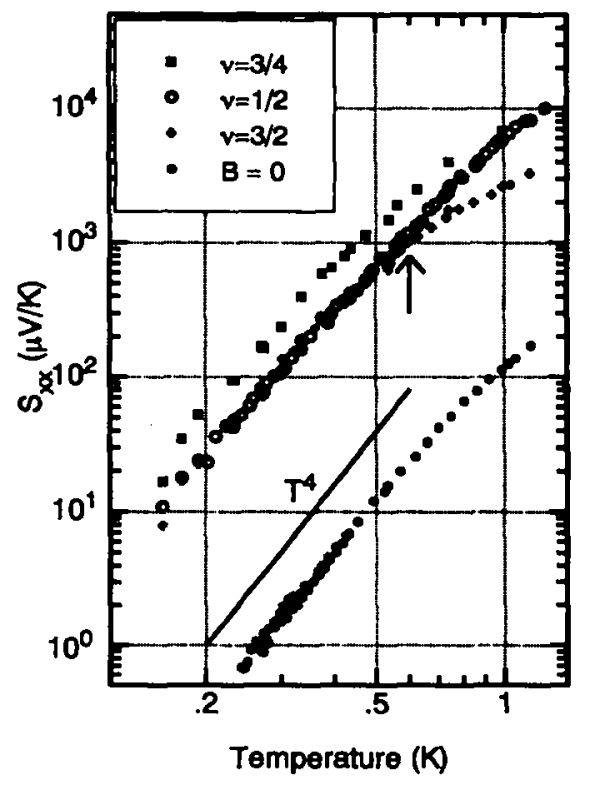

Fig. 4. The temperature dependence of $S_{x x}$ at $v=1 / 2,3 / 2$, and $3 / 4$ compared to the zero-field thermopower $S_{0}$ for $n=$ $1.8 \times 10^{15} \mathrm{~m}^{-2}$. 
field $S_{0}$ is given by $[6,9]$

$$
S g=-\frac{v L}{e T} \times \frac{m^{*}}{\tau_{\text {op }}}
$$

where $v$ is the sound velocity in the GaAs substrate, $L$ is the phonon mean free path, $m^{*}$ is the effective mass of the electrons, and $\tau_{\text {op }}^{-1}$ is the electronphonon relaxation rate. The similarity in the temperature dependences of $S_{0}$ and $S_{x x}(q / 2 m)$, where $q$ is an odd integer, suggests that the states at $v=$ $q / 2 m$ behave very much like a Fermi liquid in zero magnetic field as far as electron-phonon interaction is concerned, and that the same analysis may thus be applied to these states. This interpretation is fully in line with the picture describing particles at $q / 2 m$ filling as CFs. In this framework, the TEP amplitude is thus proportional to the product of the effective mass of a CF and the CF-phonon scattering rate $m_{\mathrm{cf}}^{*} \tau_{\mathrm{cfp}}^{-1}$, and hence this product is the same at $v=1 / 2$ and $3 / 2$. Analogously, this is also the case at $1 / 4$ and $3 / 4$, where the product is roughly two times larger. The strong enhancement of TEP of CFs compared to the zero field value $S_{8}^{8}$ can be explained partly by the enhanced effective mass of CFs compared to that of non-interacting electrons $\left(m_{\mathrm{cf}}^{*} \approx 10 m^{*}\right.$ for $v=1 / 2$, as obtained from Shubnikov-de Haas (SdH) measurements $[3,4]$ ). The remaining factor must arise from an enhanced CF-phonon coupling.

We note that the magnitude of $S_{x x}$ shows a smooth but clear maximum at $v=1 / 2$, the peak becoming more pronounced at lower temperatures and higher densities. This behaviour again differs strongly from the resistivity, where no such clear structure around $v=1 / 2$ is observed. Assuming no significant field dependence of $\tau_{\mathrm{cfp}}^{-1}$, these results suggest only a slight mass increase $(\sim 20 \%)$ around $v=1 / 2$. This observation differs from the behaviour of $m^{*}$ inferred from SdH data, which suggest a strong divergence near $v=1 / 2$ [3]. This difference might be related to the fact that an $\mathrm{SdH}$ analysis gives an effective mass for the quantum oscillations at $v$ somewhat removed from $1 / 2$, whereas in this experiment, $S_{x x}$ is sensitive to $m_{\mathrm{cf}}^{*}$ directly at $v=$ $1 / 2$ and its vicinity.

\section{Summary}

We conclude that the thermopower at filling factors $v=q / 2 m$ exhibits universal behaviour, independent of temperature and electron density. Comparison with the zero-field thermopower shows that the data are fully consistent with the picture of CFs which behave as particles in zero effective field, but with enhanced mass and electron-phonon scattering rate. We attribute the universal behaviour as being due to CFs having very similar properties with regard to their mass and phonon scattering rates. CFs with the same denominator $2 m$ appear to be very closely related.

\section{References}

[1] J.K. Jain, Adv. Phys. 41 (1992) 105.

[2] B.L. Halperin, P.A. Lee and N. Read, Phys. Rev. B 47 (1993) 7312.

[3] R.R. Du, H.L. Stormer, D.C. Tsui, A.S. Yeh, L.N. Pfeiffer and K.W. West, Phys. Rev. Lett. 73 (1994) 3274.

[4] D.R. Leadley, R.J. Nicholas, C.T. Foxon and J.J. Harris, Phys. Rev. Lett. 72 (1994) 1906.

[5] R.L. Willett, R.R. Ruel, K.W. West and L.N. Pfeiffer, Phys. Rev. Lett. 71 (1993) 3846.

[6] B. Tieke, R. Fletcher, S.A.J. Wiegers, U. Zeitler, J.C. Maan, C.T. Foxon and J.J. Harris, Physica B 211 (1995) 414; U. Zeitler, B. Tieke, S.A.J. Wiegers, J.C. Maan, R. Fletcher, V.I. Fal'ko, C.T. Foxon and J.J. Harris, in: Proceedings of the 11th International Conference on High Magnetic Fields in Semiconductor Physics (SEMMMAG-94), Cambridge, MA, USA, 1994 (World Scientific, Singapore, 1995) p. 38.

[7] U. Zeitler, J.C. Masa, P. Wyder, R. Fletcher, C.T. Foxon and J.J. Harris, Phys. Rev. B 47 (1993) 16008.

[8] X. Ying. V. Bayot, M.B. Santos and M. Shayegan, Phys. Rev. B 50 (1994) 4969.

[9] B.L. Gallagher and P.N. Butcher, in: Handbook on Semiconductors, Vol. 1, Bds. T.S. Moss and P.T. Landsberg (Elsevier, Amsterdam, 1992) p. 817. 